\title{
Benchmarking Hand and Grasp Resilience to Dynamic Loads
}

\author{
Francesca Negrello ${ }^{1}$, Werner Friedl ${ }^{3}$, Giorgio Grioli ${ }^{1}$, Manolo Garabini ${ }^{2}$, Oliver Brock ${ }^{4}$, \\ Antonio Bicchi ${ }^{1,2}$, Máximo A. Roa ${ }^{3}$, Manuel G. Catalano ${ }^{1}$
}

\begin{abstract}
In this work, we investigate the behavior of artificial hands under impulsive load conditions. Resilience to impacts has been seldom considered in grasp and manipulation literature and benchmarks, although it is one of the most relevant issues in a number of applications involving physical interactions with unstructured environments, in prosthetic as well as in robotic manipulation. We focus on two research questions: the capability of hands to withstand impacts before being damaged (Hand Resilience) and before losing the grasp on a object (Grasp Resilience). To these aims, we introduce an evaluation framework, including a precisely defined experimental set-up and test procedure. The proposed methodology, metrics, and test variables are discussed through analytical evaluation and with experimental data extracted from the testing of three different hand designs.
\end{abstract}

Index Terms-Benchmarking, hand, grasp and manipulation, mechanical design

\section{INTRODUCTION}

$\mathbf{T}$ HE aim of our study is to define a set of test methods to characterize the behavior of robotic hands under impulsive load conditions, i.e. under impacts. Our work is motivated by the fact that real-world scenarios often involve harsh and irregular physical interactions with the environment (i.e. search and rescue, craft and sports activities). Thus, the capability of robotic hands to withstand both moderate and severe impacts is a necessity for the effective deployment of reliable robotic solutions in real-world tasks, considering humanoids and bionic limbs as well [1]. These proposed test methods will accelerate the development of robotic hands for real-world applications. We focus on two research questions: 1) how to quantify physical robotic hands robustness (Hand Resilience) and 2) how an impact affects the grasp (Grasp Resilience).

In the last decade, the growing popularity of soft robotic hands has increased the end-effectors' capability to cope with

Manuscript received: August, 13, 2019; Revised October, 19, 2019; Accepted December, 3, 2019.

This paper was recommended for publication by Editor Han Ding upon evaluation of the Associate Editor and Reviewers' comments.

This work is supported by the European Union's Horizon 2020 research program under the projects SOMA (No. 645599), Soft Pro (No. 688857), and Natural Bionics (No. 810346), and by the German Research Foundation - DFG under Germany Excellence Strategy - EXC 2002/1 "Science of Intelligence" (No. 390523135). The content of this publication is the sole responsibility of the authors. The European Commission or its services cannot be held responsible for any use that may be made of the information it contains.

1 Istituto Italiano di Tecnologia, Via Morego 30, 16163 Genova, Italy, francesca.negrello@it.it.

2 Centro di Ricerca E. Piaggio e Dipartimento di Ingegneria dell'Informazione, Università di Pisa, 56126 Pisa, Italy.

3 German Aerospace Center (DLR), Institute of Robotics and Mechatronics, 82234 Wessling, Germany.

4 Robotics and Biology Laboratory, Technische Universität Berlin, 10623 Berlin, Germany.

Digital Object Identifier (DOI): see top of this page.
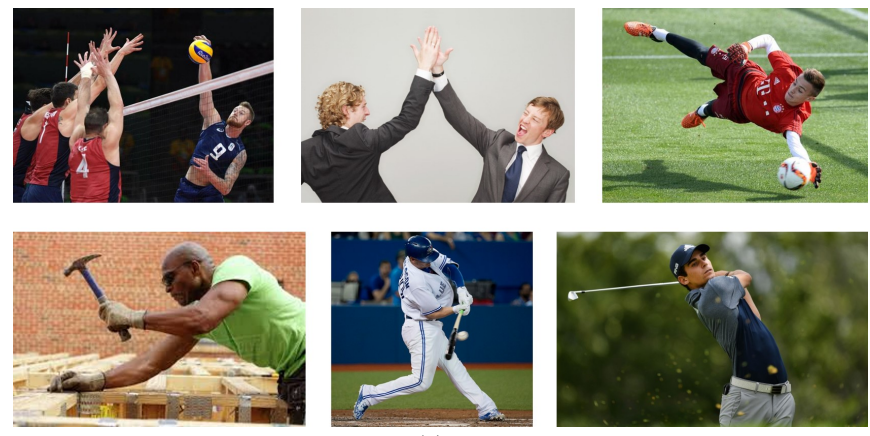

(a)

\begin{tabular}{l|c|c|c} 
& Ball mass $(\mathrm{g})$ & Max speed $(\mathrm{km} / \mathrm{h})$ & Energy $(\mathrm{J})$ \\
\hline Soccer & 450 & 180 & 562 \\
Volleyball & 280 & 132 & 188 \\
Golf & 45.93 & 349 & 215 \\
Baseball & 149 & 169 & 164 \\
Tennis & 59.4 & 263 & 158 \\
\hline
\end{tabular}

(b)

Fig. 1: (a) Examples of dynamic tasks in daily-life and sport activities involving a high energy exchange. (b) Ball mass, max speed and energy in various sports at professional level. ${ }^{1}$

physical interactions [2]. In this context, the efforts of the robotic community toward a common grasp and manipulation benchmarking framework represent one of the steps required to assess progress in the development of robotic hands [3]. Despite the advances, robotic grasp and manipulation capabilities are far from being competitive with human capabilities (Fig. 1). Furthermore, existing benchmarks mostly consider grasp stability in quasi-static conditions, and have not yet addressed dynamic interactions [3], [4], [5]. We developed a methodology for characterizing robotic hands, and a dedicated test bed for performing reproducible tests with impulsive loads. This paper extends an author's preliminary work [6] on the robotic Hands Resilience. Here we further developed the methodology, including Grasp Resilience, and extend the experimental testing to three types of soft hands technologies, including soft articulated, variable stiffness, and continuum hands. Note that although the method is applicable to any robotic hand design, we focused on soft robotic hands because, thanks to their intrinsic elasticity, the energy absorbed during the impact can be dissipated, reducing the risk of damage.

The design of the test protocol and performance evaluation is inspired by the works of Charpy and Izod for the systematic definition of resilience and toughness of materials through impact tests [7], [8]. We transfer their concepts to the realm of robotics. It is important to evaluate both the impact energy that robotic hands can withstand, and the loads

${ }^{1}$ www.guinnessworldrecords.com 
they transfer to the structure. Defining these characteristics can lead to design modifications that help avoid damage to the robot hand and supporting components (e.g. actuators, Force/Torque sensors). In the case of prosthetic applications, measuring these characteristics can also lead to designs that reduce discomfort to the user. The proposed test consists of performing repeatable impacts through a sensorized pendulum. For testing Hand Resilience, the end of the pendulum collides with the hand, while for Grasp Resilience the impact occurs on an object grasped by the hand. Through the direct measure of pendulum position and forces at the pendulum tip and the hand's base, we can evaluate both the energy absorbed during the impact and the transmissibility of the hand system.

To fully characterize Hand and Grasp Resilience, we consider a number of parameters, including whether the system is stiff or soft, the actuation type and finger couplings where the impact happens, the portion of the hand involved (i.e. one or more fingers in contact), in which direction the hand is loaded, and the size of the object. Finally, to facilitate the adaptation of the proposed testing method by other researchers, we include in the supplementary material the test protocol and a data report template. We also publish the test-bed design on the Natural Machine Motion Initiative platform ${ }^{2}$ (NMMI) [9].

The paper is organized as follows: Sec. II presents the developed framework for Resilience characterization, discussing the proposed methodology, metrics, test variables, test bench, and data representation. Sec. III-IV report the experimental results of Grasp and Hand Resilience test for the three hands tested, and discuss the effect of the main test parameters. Lastly, Sec. $\mathrm{V}$ presents the main findings and open challenges.

\section{Methodology AND Metrics}

\section{A. Methodology}

To characterize the resilience of artificial hands in dynamic tasks, two aspects should be considered: 1) how interactions affect the hand grasping capability, therefore considering the hand-tool system (Grasp Resilience), and 2) how dynamic loads affect the hand as standalone system, which corresponds to evaluating its mechanical robustness (Hand Resilience). The first point to address is how to generate reproducible impulsive loads. Several methods exist in literature to recreate dynamic load conditions, e.g. drop tower [10], crash test [11], [12], and acceleration test [13]. Taking inspiration from the Izod and Charpy toughness tests [7], we propose using an instrumented pendulum as shown in Fig. 2. This choice is motivated by the fact that it provides a testing condition close to a real impact case. Moreover, it allows to easily extract the data for the hand characterization and, thanks to its modular structure, to perform both grasp and hand resilience tests using the same setup. Finally, the test bench should be simple and affordable to reproduce in order to ease the diffusion of the benchmarking method. Fig. 2 shows the two resilience test concepts, where the robotic hand is connected to the bench structure and the pendulum generates the impulsive load. In the grasp resilience test the hand grasps an object by its geometric center and the

${ }^{2}$ www.naturalmachinemotioninitiative.com/benchmark

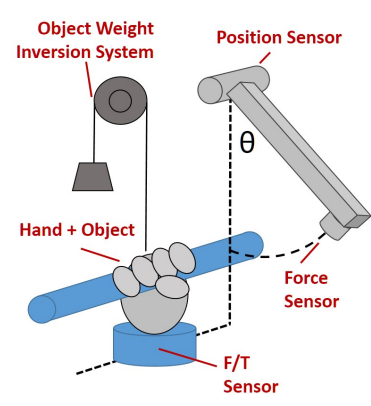

(a)

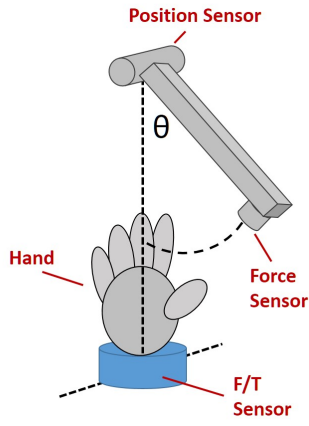

(b)
Fig. 2: Test concept for (a) Grasp Resilience, and (b) Hand Resilience.

impact happens at the object tip, while in the Hand Resilience test the pendulum directly hits the hand. The relative position between the pendulum and the hand determines the impact location, which corresponds to different load conditions. In the grasp resilience test, the object is linked to a pulley with a counterweight that reverses the gravity effects, to simulate the hand operative conditions (Fig. 2(a)).

\section{B. Metrics}

As in the Izod toughness test, we evaluate Resilience through the energy absorbed $(W)$ by the hand system during impacts. $W$ is the amount of energy required to deform a hand, or part of it, during physical interactions. Note that the capability of soft robotic hands to withstand impacts (Resilience) depends on their ability to deform elastically. Therefore, Resilience is inversely proportional to $W$. A high value of $W$ indicates a large energy exchange between the hand system and the environment. Another relevant parameter that characterizes a robotic hand system is load transmissibility, which defines the portion of the external loads that are effectively transferred through the system. Finally, it is important to measure the maximum impact a system can survive, which is defined by testing the robotic hand up to its failure point. In summary, the proposed parameters are:

- Absorbed Energy (W): difference between the energy of the pendulum before and after the impact.

- System Transmissibility (Tr): ratio between the force at the impact and at the base.

- Energy for system failure: energetic level that causes failure, either by damage to the hand or by grasp failure.

\section{Test Variables}

Here we discuss the effect of the main test parameters in order to define a precise test procedure and identify the most relevant data to collect for benchmarking both Grasp and Hand Resilience.

1) Pendulum Energy $\left(E_{0}\right)$ : defines the severity of the test. $E_{0}$ depends both on the pendulum starting position ( $\theta$ in Fig. 2) and pendulum properties (mass and length). Although we could in principle vary only one of those, during the experiments we tuned both $\theta$ and the pendulum mass to span 


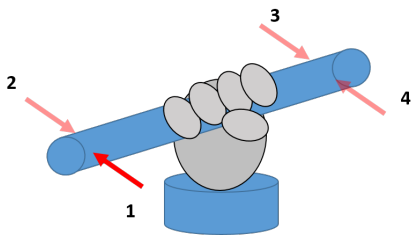

(a)

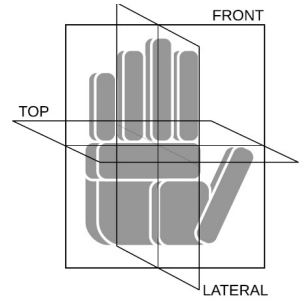

(b)
Fig. 3: Hand/load orientation for (a) Grasp and (b) Hand Resilience tests. The numbers in (a) label the different configurations tested.

a larger energetic range. The pendulum speed at the impact point is directly proportional to $E_{0}$; during the test it ranged up to $6 \mathrm{~m} / \mathrm{s}$. It is worth noticing that the maximum end-effector speed of collaborative robots ${ }^{3}$ usually does not exceed $2 \mathrm{~m} / \mathrm{s}$.

2) Hand/Load relative Orientation: An important parameter of the test is the orientation between the load and the hand. As shown in Fig. 3(a), for an item grasped by its geometric center four different load configurations are possible. Grasp resilience is strongly affected by the morphology of the end-effector. In anthropomorphic hands, the thumb position determines a variation of grasp resilience as function of hand/load orientation, while symmetric hands are less affected. Concerning hand resilience, the hand behavior under dynamic loads strongly depends on its joint design, e.g. pin, full elastic coupling, selectively compliant, etc; [14] reviews the main joint designs in literature. Consequently, testing different orientations between hand and external load is of the utmost importance in order to characterize its resilience. Due to the different hand morphologies it is not trivial to identify a priori the principal directions for any hand. Therefore, we choose as convention the anatomical planes for the hand, as shown in Fig. 3(b). In this work we tested the hands along the frontal and lateral planes. Note that the proposed test bench (Sec. II-D) does not allow to produce an impulsive load normal to the top plane; this would require a different setup, e.g. a system to impose a specific hand displacement.

3) Contact Area: For each hand orientation it is possible to identify different contact areas, i.e. what portions of the hand are effectively in contact. This is fundamental when considering underacted hands that rely on differential transmission ([9], [15], [16], [17], [18]). In this study, we considered the impact cases shown in Fig. 4 and labeled with the letters (a) to (k). Note that in the frontal plane it is possible to identify different conditions, e.g. impact happening at the fingertip (distal) or at the base (proximal). To consider this factor, the hands were tested both at $2 / 3$ of the finger length (cases a-d) and at $1 / 3$ of the finger length (cases e-h).

4) Stiffness: Hand stiffness can be actively changed in the case of VSA mechanisms [15], by varying the pressure of a fluidic actuation system [19], or by passively changing the finger elastic properties. Stiffness preset is an important parameter that affects both grasp and hand resilience. Grasp stiffness is closely related to the grasp strength: a stiffer grasp

\footnotetext{
${ }^{3}$ www.universal-robots.com, www.franka.de, www.kuka.com
}

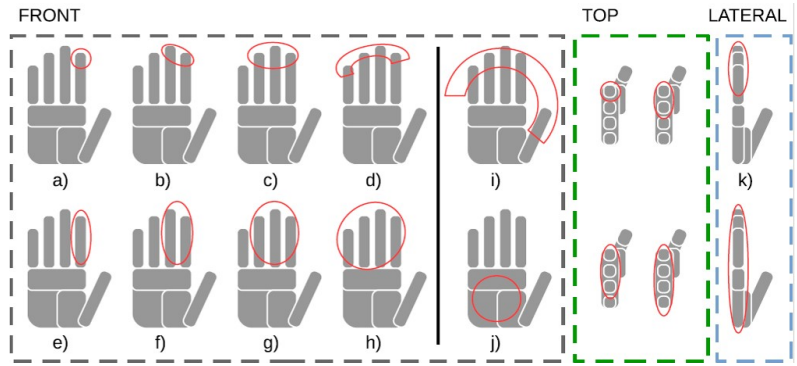

Fig. 4: Examples of contact conditions for an anthropomorphic hand.

is usually more stable. At the same time, a higher stiffness corresponds to a higher absorbed energy, with a consequent higher risk of damaging the hand in case of collisions.

5) Object Size and Shape: Provided that artificial hands have different size and closure envelopes, the object size and shape play a relevant role in determining Grasp Resilience. In this work we prioritized the effect of the object size, testing 3 cylindrical objects with different diameters $(2-4-6 \mathrm{~cm})$, and fixed length $(30 \mathrm{~cm})$.

\section{Experimental Test-bench}

The test setup is based on a sensorized pendulum, as presented in [6], that generates repeatable impacts (Fig. 5). Oscillations are measured by a magnetic encoder located on the pendulum joint (AS5045), an uniaxial force sensor on the pendulum tip measures the impulsive force (Dytran 1051V4), and a 6 axis Force/Torque sensor (ATI OMEGA160) at the hand base measures the forces transferred to the structure after the perturbation. Pendulum angular position is sampled at $250 \mathrm{~Hz}$, while the impulsive forces are sampled at $10 \mathrm{kHz}$ through a DAQ system (NI-6251). The structure is designed to be fully modular and allows both vertical and horizontal settings. Specific contact conditions (e.g. number of phalanxes) are obtained by changing the pendulum striker. We used 3D printed strikers to easily adapt the system to the different hand sizes tested. When testing stiff hands, the striker stiffness may affect the results, and a steel striker is preferable in that case.

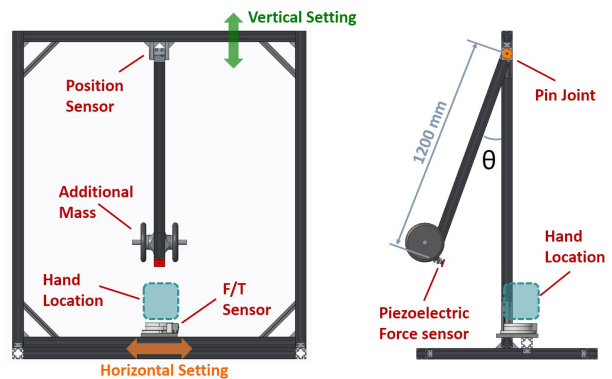

Fig. 5: CAD model of the experimental setup, front and side view.

\section{E. Data representation}

During the resilience experiments we recorded the pendulum angular position and the force at the pendulum tip (impact head) and hand base. Figs. 6-10 illustrate how we represent 
the data to extract the proposed metrics. Specifically, the energy absorbed is evaluated by comparing the initial and final pendulum energy, which is directly proportional to its angular position (Fig. 6). Through the evaluation of the pendulum phase plot it is possible to visualize the energy absorption as a step between different iso-energy curves. Transmissibility, instead, is evaluated as the ratio between the peak force measured at the hand base and the peak force measured at the impact head, $\alpha F / F$ (Fig. 7). The shift in time of the peak force is due to the delay introduced by the load transmission in the structure.

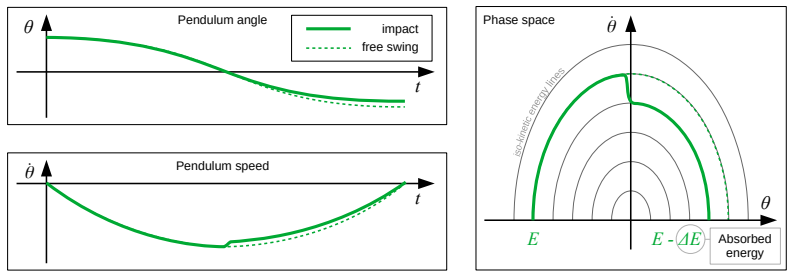

Fig. 6: Examples of pendulum position and speed vs. time (left) and pendulum phase space (right). The impact causes a curve shift from the free swing case, which in the phase space is visualized as two different energetic levels.

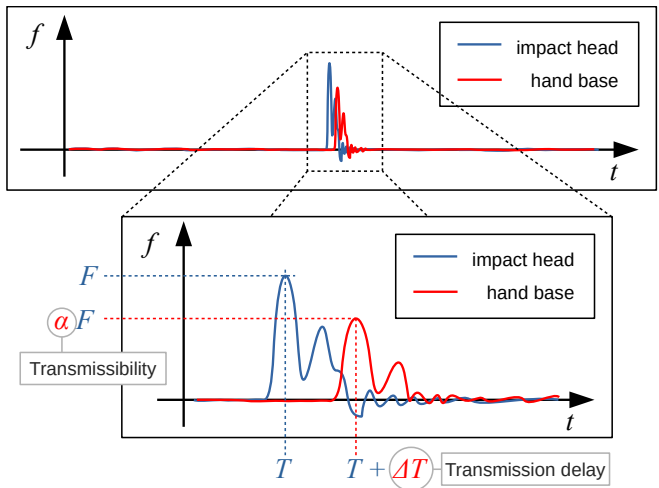

Fig. 7: Example of force vs time plot; the detailed view highlights the extraction of transmissibility data.

To represent energy absorption and transmissibility as function of the test parameters, e.g. number of fingers in contact (Fig. 8), we used box-plots where the continuous line links the mean values, and the whiskers show data variation over the test repetitions. As already discussed, the Resilience test spans a large number of parameters, which identify the configuration space (Fig. 9). Each point of the configuration space corresponds to an experiment, and is related to a value of absorbed energy, transmissibility, success rate, and object misalignment. For the data representation we use tables that correspond to the extraction of a subset of the configuration space. Fig. 10 shows the definition of the color scale for Grasp Resilience. A grasp is successful if after the impact the object is still stably grasped and there is no variation of the object initial orientation. We consider that a condition with $50 \%$ failure rate does not provide a grasp that can be defined as stable, and therefore this determines the limit of the grasp resilience (failed grasp). Between the successful and failure

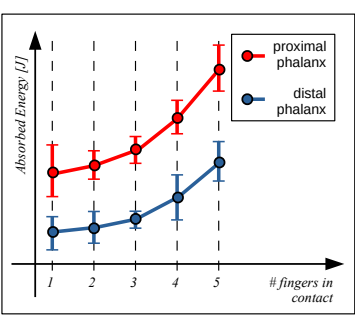

(a)

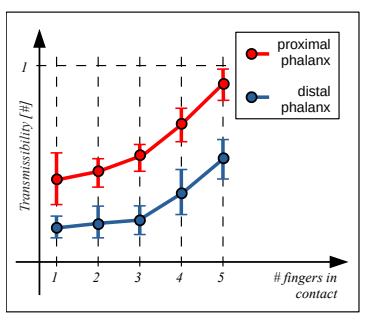

(b)
Fig. 8: Examples representing Energy absorption and Transmissibility, whiskers show data variance over repetitions. Transmissibility of 1 means that the system transfers the full impact load to its base, a value smaller than one shows a load attenuation, while transmissibility above one would indicate an amplification of the load.

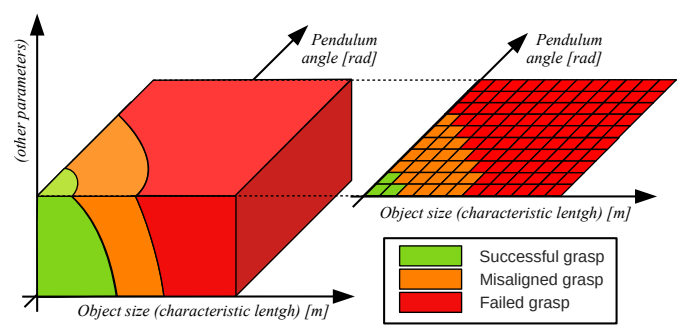

Fig. 9: Example of configuration space representation and table extraction.

grasp exists an intermediate orange zone, which corresponds to those configurations that after the impact show an object misalignment and have a failure rate below 50\%. We assume that this grasp resilience confidence level is defined by the user's application, therefore the acceptable subset of the grasp resilience map should be evaluated for each specific case.

\section{EXPERIMENTAL CHARACTERIZATION: GRASP RESILIENCE}

Experimental tests were performed on three robotic hands, which are representative of different soft technologies: Pisa/IIT SoftHand [9], CLASH hand [15], RBO 2 hand [19], whose specifications are reported in the Appendix. In the remainder of the paper those are referred to as $\mathrm{SH}, \mathrm{CH}$ and $\mathrm{RBO}$,
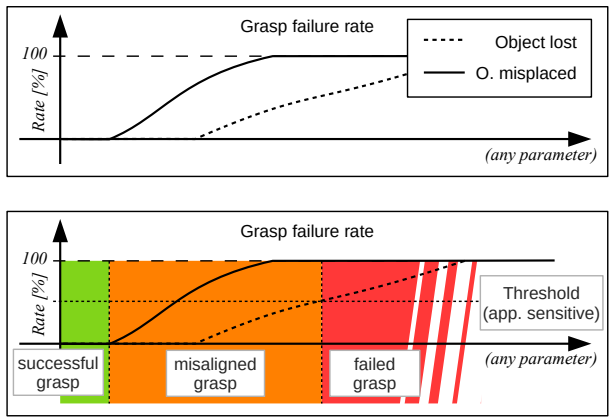

Fig. 10: Example of Grasp failure rate and object misalignment color scale. 
respectively. Here, we report the results of the experimental characterization of these soft robotic hands as an application example of the proposed benchmark, and to show the effect of the main test parameters. The complete set of results of the Grasp Resilience characterization of each hand are reported in the supplementary material.

Pendulum Energy: Figs. 11-12 show the effect of pendulum initial energy over $\mathbf{W}$ and $\mathbf{T r}$ for different hand configurations. While $\mathbf{W}$ is proportional to $E_{0}$, transmissibility is constant, with an experimental variation within $5 \%$. Note that larger changes in Tr happen when the grasp fails or is unstable due to the load condition (e.g. Fig. 11(a) Config. 3 from 60 deg to 90 deg). Note that each configuration was tested up to its failure point, therefore they show a different number of data points.

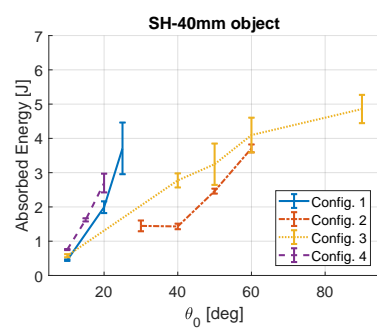

(a)

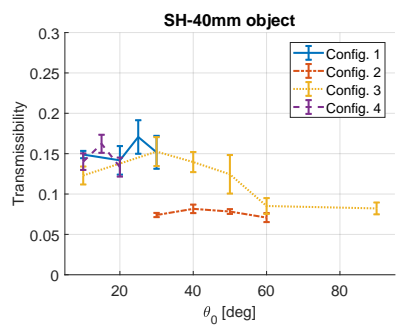

(b)
Fig. 11: Effect of pendulum initial energy $\left(\theta_{0}\right)$ over (a) $\mathbf{W}$ and (b) $\mathbf{T r}$ for different hand configurations in the $\mathrm{SH}$.

Hand/Load Orientation: the effect of the hand/load orientation can be observed in Fig. 11, where to each configuration corresponds a different value of $\mathbf{W}$ and $\mathbf{T r}$. Moreover, the color maps (Figs. 15-16) provide an overview of the grasp success rate under a specific load for each hand/load configuration and pendulum initial energy (release angle).

Grasp Stiffness: Fig. 13 shows the effect of a variation of grasp stiffness over transmissibility and energy absorption. Intuitively, to a stiffer hand corresponds a higher $\mathbf{W}$ and $\mathbf{T r}$, and its grasp is more stable (Fig. 14).

Object Size and Shape: Fig. 12 shows the effect of object size on the SH absorbed energy (a) and transmissibility (b). It is worth considering that for the $60 \mathrm{~mm}$ object the grasp fails at a low energy level, while the grasp of smaller objects is stable also at higher energy levels. The object size also affects the overall absorbed energy. Fig. 13 shows the effect of

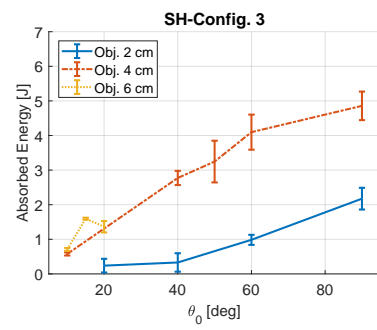

(a)

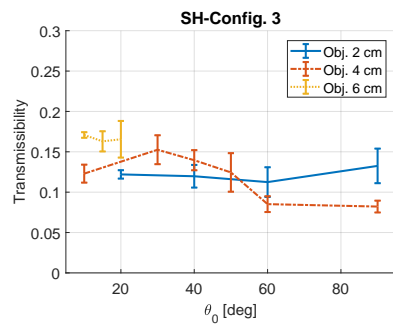

(b)
Fig. 12: Effect of pendulum initial energy $\left(\theta_{0}\right)$ over (a) $\mathbf{W}$ and (b) $\mathbf{T r}$ for different object sizes in the $\mathrm{SH}$.

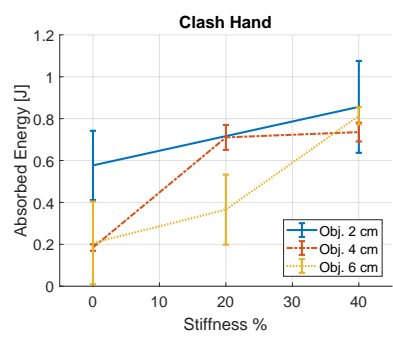

(a)

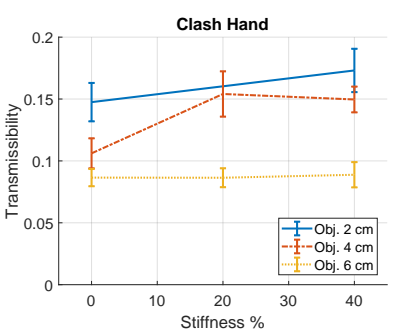

(b)
Fig. 13: Effect of grasp stiffness over (a) $\mathbf{W}$ and (b) $\mathbf{T r}$ for different object sizes in the $\mathrm{CH}$ (Config. 1).

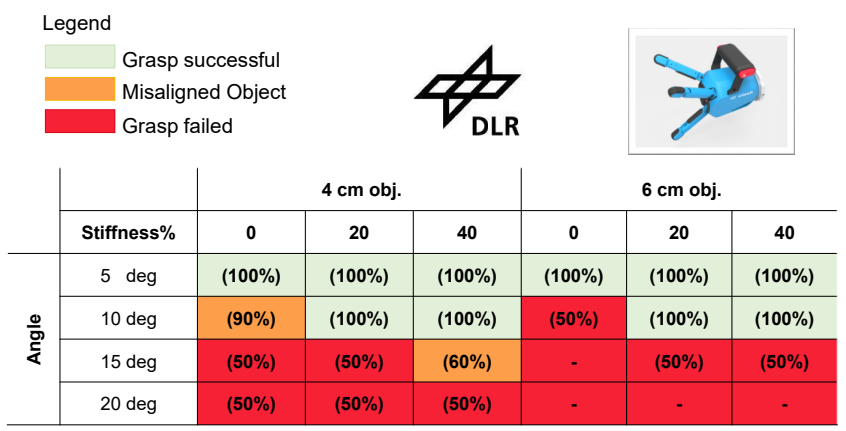

Fig. 14: Example of grasp resilience map for the CLASH Hand.

object size on CH absorbed energy (a) and transmissibility (b). Note that stiffening the hand, its deformability is reduced and consequently the energy absorbed is less affected by the object size. In the same way, grasping a large object requires a higher force, thus reducing the transmissibility variation.

\section{Discussion}

One of the results of this work is the characterization of three robotic hands, whose full data is reported in the additional material. Comparing the different hands design, a few considerations should be done. The first one concerns the end-effector morphology, as grasp resilience is strongly affected by thumb location. In particular, both RBO and $\mathrm{SH}$ show Config. 3 as the best orientation (Fig. 15-16). At the same, time finger size and location does affect the size range of the graspable objects. In this work we used cylindrical objects with steps of $2 \mathrm{~cm}$ on diameter (2-4-6-8 $\mathrm{cm})$. During the tests we observed that the cylinder of $2 \mathrm{~cm}$ diameter was too small to obtain a stable grasp with the $\mathrm{RBO}$ and $\mathrm{CH}$, while the $8 \mathrm{~cm}$ one was too large. This raises a possible issue related to the large number of tests to cover the largest range of object sizes. A possible way to reduce the number of experiments to characterize a hand is to use normalized objects with respect to the hand size. Secondly, it is worth noticing that both $\mathrm{SH}$ and $\mathrm{RBO}$ had compliance at the hand base, while the $\mathrm{CH}$ was rigidly connected to the structure. We think that this additional compliance determined the extended grasp resilience of RBO and $\mathrm{SH}$ with respect to $\mathrm{CH}$.

The results of the presented work on Grasp Resilience also open some questions for future work. Among others, we highlight the presence of an orange zone in the color 


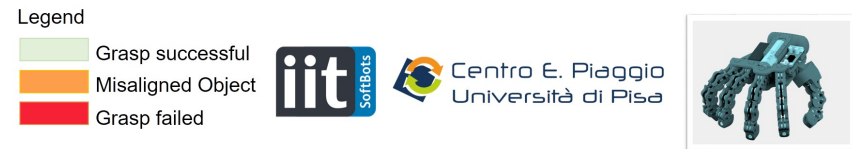

Config. 1

Config. 4

\begin{tabular}{|c|c|c|c|}
\hline Angle & $2 \mathrm{~cm}$ obj. & $4 \mathrm{~cm}$ obj. & $6 \mathrm{~cm}$ obj. \\
\hline $5 \mathrm{deg}$ & - & - & $(100 \%)$ \\
\hline $10 \mathrm{deg}$ & $(100 \%)$ & $(100 \%)$ & $(100 \%)$ \\
\hline $15 \mathrm{deg}$ & - & - & $(90 \%)$ \\
\hline $20 \mathrm{deg}$ & $(90 \%)$ & (90\%) & $(50 \%)$ \\
\hline $25 \mathrm{deg}$ & $(80 \%)$ & $(80 \%)$ & - \\
\hline $30 \mathrm{deg}$ & $(50 \%)$ & $(30 \%)$ & - \\
\hline
\end{tabular}

Config. 2

\begin{tabular}{l|c|c|c}
\hline \multicolumn{1}{l}{ Angle } & $2 \mathrm{~cm}$ obj. & $4 \mathrm{~cm}$ obj. & $6 \mathrm{~cm}$ obj. \\
\hline $5 \mathrm{deg}$ & - & - & $(\mathbf{1 0 0 \% )}$ \\
\hline $10 \mathrm{deg}$ & - & $(100 \%)$ & $(\mathbf{7 0} \%)$ \\
\hline $15 \mathrm{deg}$ & - & - & $(50 \%)$ \\
\hline $20 \mathrm{deg}$ & - & - & $(50 \%)$ \\
\hline $30 \mathrm{deg}$ & $(100 \%)$ & $(100 \%)$ & - \\
\hline $40 \mathrm{deg}$ & $(100 \%)$ & $(100 \%)$ & - \\
\hline $50 \mathrm{deg}$ & - & $(100 \%)$ & - \\
\hline $60 \mathrm{deg}$ & - & $(50 \%)$ & - \\
\hline $90 \mathrm{deg}$ & $(100 \%)$ & $(50 \%)$ & - \\
\hline
\end{tabular}

\begin{tabular}{c|c|c|c}
\hline \multicolumn{1}{l}{ Angle } & $2 \mathrm{~cm}$ obj. & \multicolumn{4}{c}{ cm obj. } & $6 \mathrm{~cm}$ obj. \\
\hline 5 deg & - & - & $(100 \%)$ \\
\hline $10 \mathrm{deg}$ & - & $(100 \%)$ & $(50 \%)$ \\
\hline $15 \mathrm{deg}$ & - & $(100 \%)$ & - \\
\hline $20 \mathrm{deg}$ & $(100 \%)$ & $(40 \%)$ & - \\
\hline $30 \mathrm{deg}$ & $(100 \%)$ & - & - \\
\hline 40 deg & $(50 \%)$ & - & - \\
\hline
\end{tabular}

Config. 3

\begin{tabular}{l|c|c|c}
\hline \multicolumn{1}{l}{ Angle } & 2 cm obj. & 4 cm obj. & 6 cm obj. \\
\hline 5 deg & - & - & - \\
\hline 10 deg & - & $(100 \%)$ & $(100 \%)$ \\
\hline 15 deg & - & - & $(50 \%)$ \\
\hline 20 deg & $(100 \%)$ & - & $(50 \%)$ \\
\hline $30 \operatorname{deg}$ & - & $(80 \%)$ & - \\
\hline $40 \operatorname{deg}$ & $(100 \%)$ & $(100 \%)$ & - \\
\hline 50 deg & - & $(70 \%)$ & - \\
\hline 60 deg & $(100 \%)$ & $(70 \%)$ & - \\
\hline 90 deg & $(100 \%)$ & $(60 \%)$ & - \\
\hline
\end{tabular}

Fig. 15: Example of grasp resilience map for the Pisa/IIT SoftHand.
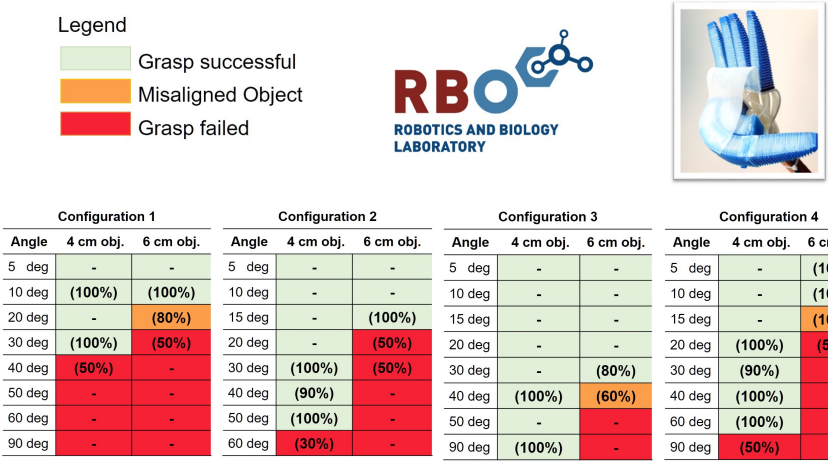

Configuration 4 Angle $4 \mathrm{~cm} \mathrm{obj} .6 \mathrm{~cm} \mathrm{obj}$. \begin{tabular}{|c|c|c|}
\hline $5 \mathrm{deg}$ & - & $(100 \%)$ \\
\hline $10 \mathrm{deg}$ & - & $(100 \%)$ \\
\hline $15 \mathrm{deg}$ & - & $(100 \%)$ \\
\hline
\end{tabular} $-(100 \%)$ $(100 \%)$ \begin{tabular}{r|r}
\hline $20 \mathrm{deg}$ & $(100 \%)$ \\
\hline $30 \mathrm{deg}$ & $(90 \%)$
\end{tabular} \begin{tabular}{l|l}
\hline 30 deg & $(90 \%)$ \\
\hline $40 \mathrm{cos}$ & $(100 \%)$ \\
\hline
\end{tabular}

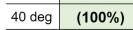
$(100 \%)$ $90 \mathrm{deg}$

Fig. 16: Example of grasp resilience map for the RBO Hand 2.

maps (Figs. 14, 15, 16), which in presence of a high success rate, denotes a strong variation of object orientation (Fig. 17), that could affect the manipulation task the robot is executing. Therefore, it seems relevant to investigate the capability of a manipulator to cope with certain degrees of uncertainty in object orientation, to identify what is the maximum acceptable misalignment. Another aspect worth investigating is the correlation between Hand and Grasp Resilience, looking toward a unified color map that defines the range in which the grasp would fail without consequences on the hardware, and those impact conditions that would damage it. Finally, the Grasp Resilience setup could be used to characterize the artificial hand's center of pressure, which could be beneficial for prosthesis design and testing, or for testing sport equipment.

\section{EXPERIMENTAL CHARACTERIZATION: HAND RESILIENCE}

This section reports the experimental results of hand resilience tests over the three robotic hands considered (for

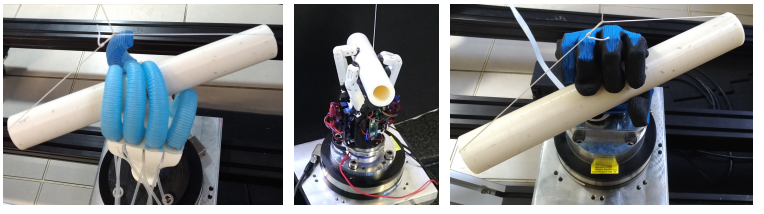

Fig. 17: Examples of object misalignment after an impact.

specifications refer to the Appendix), and shows the effect of the main test parameters. The complete Hand Resilience characterization for each hand is reported as additional material.

Pendulum Energy: as Fig. 18 shows, increasing $E_{0}$ the energy absorption among different impact conditions becomes less relevant. As discussed in a previous work [6], this saturation-like effect defines the maximum energy a compliant hand absorbs under dynamic loads. This value is particularly relevant for designing hands that withstand dynamic loads.

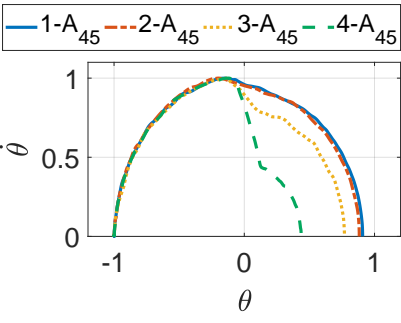

(a)

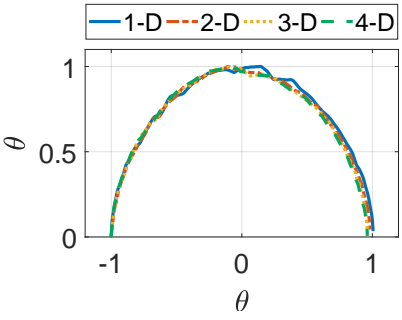

(b)
Fig. 18: Experimental phase plots showing the effect of (a) different $E_{0}$ specifications, and (b) different number of fingers in contact, as indicated in the legend.

Hand Orientation: Fig. 19 shows the energy absorbed by a finger loaded from different directions. Intuitively, the energy absorbed increases with the stiffness preset. Due to its transmission design, the $\mathrm{CH}$ finger shows an asymmetric stiffness profile for the different directions. The front one shows the larger variation and it absorbs up to $3 J$ at $80 \%$ stiffness, followed by lateral and dorsal direction. Considering the lateral direction, the free moving range is $\pm 20^{\circ}$ (ab/ad), if an impact happens the finger can dislocate after $20^{\circ}$ from is joint sleeve to sidestep the collision. This motion consumes much more spring range as the $20^{\circ}$ before, due to the increase in lever arm (the rotation center changes from middle to left or right bearing of the cardan joint) mostly noticeable at the base extensor, which is most dominant for stiffness variation. A stiffness preset shortens the lateral spring range of motion, resulting in a stiffer joint. In this case the failure point is about $2 J$ at $20 \%$ stiffness (Fig. 19(a)). Finally, it is worth mentioning the effect of the surface on the absorbed energy, as there is a large variation between the polylactide surface at the dorsal side and the rubber finger pads in the frontal side.

Contact Area: Fig. 20 shows transmissibility and absorbed energy of SH for both distal (blue) and proximal (red) impact condition, considering up to 4 fingers in contact. As expected, increasing the portion of the hand in contact (number of fingers and number of phalanxes) corresponds to a higher 


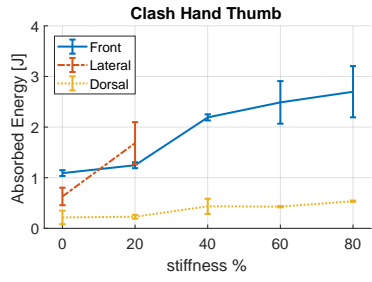

(a) (b)

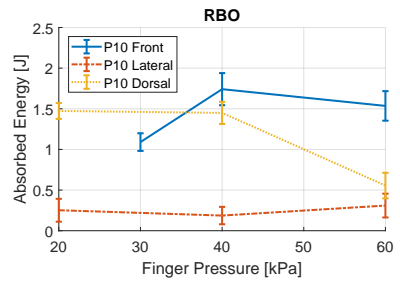

Fig. 19: Effect of a variation in the direction of the impact on (a) the CLASH hand thumb, and (b) the RBO finger.

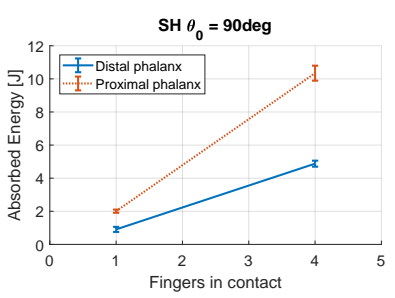

(a)

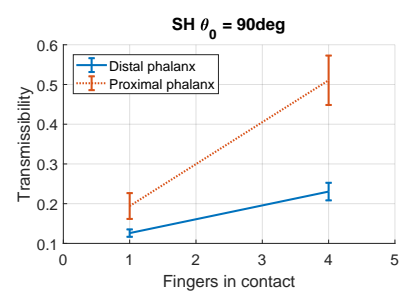

(b)
Fig. 20: Effect of contact area on absorbed energy and transmissibility. The blue line corresponds to the (a) and (d) cases, while the red line spans the (e) to (h) cases (Cases are defined in Fig. 4).

hand stiffness and inertia, with a subsequent effect on $\mathbf{W}$ and $\mathbf{T r}$. The toughest condition is (h), which corresponds to $10 \mathrm{~J}$ with transmissibility about 0.5 . Fig. 21 shows the difference between an impact involving one of the two coupled fingers (underactuated) or both of them (fully coupled) in $\mathrm{CH}$. In the second case the hand is much more stiff, and at $40 \%$ stiffness reaches the failure point above $3 J$.

Stiffness: Fig. 21 shows the energy absorption and transmissibility trend of the $\mathrm{CH}$ as function of its stiffness. A peculiar characteristic of the fluidic continuum systems like the RBO, is the effect of source pressure, which affects both finger stiffness and position (curvature), as shown in Fig. 22. As a matter of fact, increasing the pressure causes both finger stiffening and bending, with the consequent change of orientation of the fingertip with respect to the pendulum striker. Consequently, above $40 \mathrm{kPa}$ the absorbed energy for the finger on the front and dorsal direction decreases (Fig. 19(b)), while the lateral direction is less affected by the finger deflection.

\section{Discussion}

The main result of the experimental validation is the hands' characterization, which is reported in the additional material. In particular, through the use of the color maps it is possible to define a safe operation range for a manipulator subject to dynamic loads. Introducing compliance in the hand design strongly influences both its energy absorption and transmissibility, consequently increasing its resilience. Comparing the three hands, RBO has the lowest energy absorption, thanks to its high deformability and low finger inertia. At the same time, SH withstands high energy levels, thanks to the differential transmission through the fingers (underactuation) and their elastic joint design. Lastly, the test allows to do failure anal-

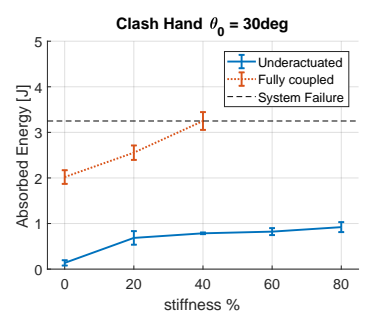

(a)

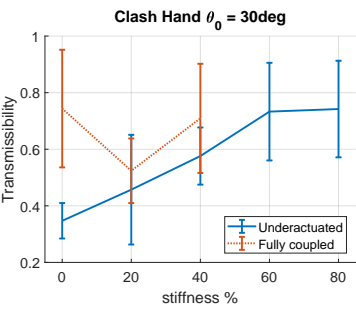

(b)
Fig. 21: Effect of variation in finger stiffness during impact on (a) energy absortion, and (b) transmissiblity. It is worth noticing the effect of the transmission design comparing the two curves in each plot, blue corresponds to the underactuated configuration (one coupled finger) and red to the fully coupled one (both coupled fingers).
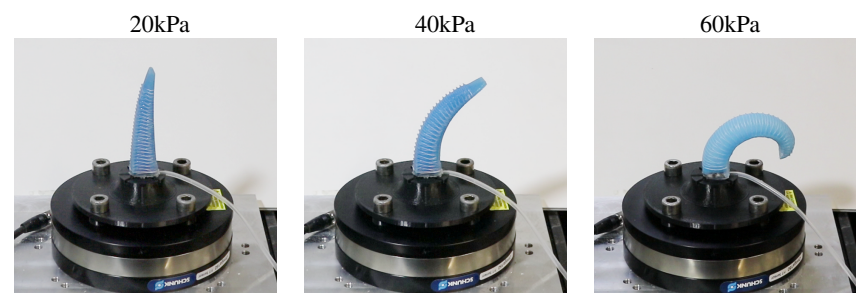

Fig. 22: RBO pneumatic finger curvature at different pressure levels.

ysis, highlighting the components that are more likely to fail under dynamic loads. For instance, we observed that typical failure for tendon-driven hands is at the tendon terminals $(\mathrm{CH})$. Another component that should be designed properly is the elastic connection at the hand base: while it allows to dissipate energy, it also represents a possible failure point, being subject to bending and torsional loads ( $\mathrm{SH})$. Finally, the main issues of silicon-based continuum hands (RBO) are leakages due to overpressure in the ducts, and the strength of the bonding between silicon (finger structure) and stiff parts that are used to assemble the different hand elements.

\section{CONCLUSIONS}

We proposed a method for characterizing and benchmarking the resilience of robotic hands under dynamic loads. The method considers grasp capability and physical sturdiness of the hand. Our study investigated the main parameters of the test that affect the grasp and hand resilience, and identified those that allow the improvement of resilience. Moreover, we provided a precise protocol and benchmark template to enable standardization and reproducibility of the test. Based on the proposed characterization, we reported the grasp and hand resilience of three soft robotic hands. Soft hands are much more likely to withstand the impact test, whereas noncompliant hands run the risk of serious damage.

Our results are relevant for a number of real-world applications. For instance, in the field of collaborative robotics, hand and grasp resilience could be used to define the safe operating conditions for a manipulator as a function of its velocity and inertia. To improve the setup, one could include mechanical fuses to prevent too high energy transfer and to render the testing of rigid hands less destructive. Future work will investigate if the planes selected for analysis correspond 


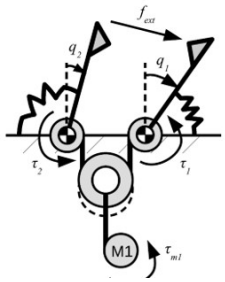

(a)

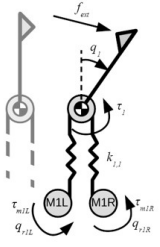

(b)

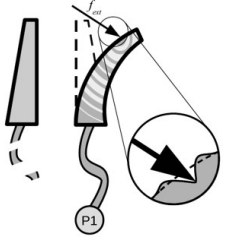

(c)
Fig. 23: Tested soft hand technologies: (a) $\mathrm{SH}$, (b) $\mathrm{CH}$, (c) RBO.

to the principal components of the hand. In that case, any other hand/load orientation could be represented as a combination of these principal components. Moreover, we will explore the correlation between hand and grasp resilience toward a unified resilience map.

\section{ACKNOWLEDGMENT}

The authors would like to thank Mattia Poggiani for his support for developing the acquisition system, Steffen Puhlmann and Vincent Wall for their support testing the RBO hand.

\section{APPENDIX I - HANDS SPECIFICATIONS}

Pisa/IIT SoftHand (SH): it is an underactuated hand with 19 DoF, distributed in an anthropomorphic structure and a single actuation unit (DoA), Fig. 23(a). The soft joint design of its finger phalanxes is based on the rolamite concept [20], which enables them to survive severe disarticulations [9].

CLASH Hand (Compliant Low cost Antagonistic Servo Hand): it is a 3-finger hand based on a modular design using off-the-shelf low cost components [15]. The main feature of the hand is the variable stiffness (Fig. 23(b)) that allows it to withstand collisions with the environment, and to adapt the passive stiffness to the object weight.

RBO Hand 2: it is an anthropomorphic soft continuum hand [19], Fig. 23(c). Five actuators make up the hand's five fingers; two additional actuators permit opposing the thumb. The entirely soft design provides a large number of passive degrees of freedom and shape adaptability that allow the exploitation of environmental constraints [21].

Table I reports the main specifications of these robotic hands.

TABLE I: Specifications for soft robotic hands, from [9], [15], [19].

\begin{tabular}{|c|c|c|c|c|}
\hline \multicolumn{2}{|l|}{ Specifications } & SH & CH & RBO \\
\hline \multirow{4}{*}{ Size [22] } & a (mm) & 207 & 230 & 170 \\
\hline & $\mathrm{b}(\mathrm{mm})$ & 80 & 85 & 85 \\
\hline & $\mathrm{c}(\mathrm{mm})$ & 91 & 100 & 91 \\
\hline & $\mathrm{d}(\mathrm{mm})$ & 13.5 & 21 & 20 \\
\hline \multirow{2}{*}{ Kinematics } & DoF & 19 & 7 & $\infty$ \\
\hline & DoA & 1 & 8 & 7 \\
\hline \multirow{2}{*}{ Material } & Type & Plastimid 6 & PA-PLA & Dragonskin-PA \\
\hline & $\sigma$ yield (MPa) & 165 & $42-45$ & 3.3-48 \\
\hline \multirow[t]{2}{*}{ Weight } & Full $(\mathrm{kg})$ & 0.5 & 0.65 & $0.25^{*}$ \\
\hline & Joint $(\mathrm{N} / \mathrm{m})$ & 600 & $10-2000$ & N/A \\
\hline Linear Stiffness & Base Damper $(\mathrm{N} / \mathrm{m})$ & 1860 & - & - \\
\hline \multirow{3}{*}{ Joint Mobility } & Flexion/Extension & $-180+90 \mathrm{deg}$ & $\pm 90 \mathrm{deg}$ & $\pm 180 \mathrm{deg}$ \\
\hline & Lateral bend & $\pm 90 \mathrm{deg}$ & $\pm 90 \mathrm{deg}$ & $\pm 180 \mathrm{deg}$ \\
\hline & Twist & $\pm 180 \mathrm{deg}$ & - & $\pm 180 \mathrm{deg}$ \\
\hline
\end{tabular}

APPENDIX II - INDEX TO SUPPLEMENTARY MATERIAL

Table II reports the supplementary material repositories.
TABLE II: Supplementary material type, description and repository.

\begin{tabular}{ccll}
\hline$\#$ & Media Type & Description & Repository \\
\hline 1 & Document & Resilience benchmark description and protocol & Multimedia material \\
2 & Document & Resilience characterization for SH, CH and RBO & Multimedia material \\
3 & Document & Resilience benchmark editable template & NMMI web-site \\
4 & CAD/Software & Open source CAD model and software of the test-bench & NMMI web-site \\
5 & Video & Video of the experimental test execution & Multimedia material \\
\hline+ https://www.naturalmachinemotioninitiative.com/benchmark &
\end{tabular}

\section{REFERENCES}

[1] A. Billard and D. Kragic, "Trends and challenges in robot manipulation," Science, vol. 364, no. 6446, 2019.

[2] M. Bonilla, E. Farnioli, C. Piazza, M. Catalano, G. Grioli, M. Garabini, M. Gabiccini, and A. Bicchi, "Grasping with soft hands," in IEEE-RAS Int. Conf. Humanoid Robots, 2014, pp. 581-587.

[3] J. Falco, K. V. Wyk, S. Liu, and S. Carpin, "Grasping the performance: Facilitating replicable performance measures via benchmarking and standardized methodologies," IEEE Robotics \& Automation Magazine, vol. 22, no. 4, pp. 125-136, Dec 2015.

[4] Y. Sun, J. Falco, N. Cheng, H. Choi, E. Engeberg, N. Pollard, M. A. Roa, and Z. Xia, "Robotic grasping and manipulation competition: Task pool," in Robotic Grasping and Manipulation: First Robotic Grasping and Manipulation Challenge. Springer, 2016, pp. 1-18.

[5] B. Calli, A. Walsman, A. Singh, S. Srinivasa, P. Abbeel, and A. M. Dollar, "Benchmarking in manipulation research: Using the Yale-CMUBerkeley object and model set," IEEE Robotics \& Automation Magazine, vol. 22, no. 3, pp. 36-52, Sep. 2015.

[6] F. Negrello, M. Catalano, M. Garabini, G. Grioli, N. Tsagarakis, and A. Bicchi, "Benchmarking resilience of artificial hands," in IEEE Int. Conf. on Robotics and Automation (ICRA), 2019, pp. 8374-8380.

[7] "ISO 148-1 metallic materials - Charpy pendulum impact test — part 1: Test method," 2016.

[8] "UNI EN ISO 180:2013 - Plastics - determination of Izod impact strength." 2013.

[9] C. Della Santina, C. Piazza, G. Gasparri, M. Bonilla, M. Catalano, G. Grioli, M. Garabini, and A. Bicchi, "The quest for natural machine motion: An open platform to fast-prototyping articulated soft robots," IEEE Robotics \& Automation Magazine, vol. 24, no. 1, pp. 48-56, 2017.

[10] J. Joyce and E. Hackett, "An advanced procedure for J-R curve testing using a drop tower," in Nonlinear Fracture Mechanics, vol. I, 1988, pp. 289-317.

[11] S. Haddadin, A. Albu-Schäffer, and G. Hirzinger, "Safety evaluation of physical human-robot interaction via crash-testing," in Robotics: Science and Systems III, 2007, pp. 217-224.

[12] M. Segui-Gomez, F. Lopez-Valdes, and R. Frampton, "An evaluation of the EuroNCAP crash test safety ratings in the real world," in Annual Proc. Assoc. Adv. of Automotive Medicine, vol. 51, 2007, pp. 281-298.

[13] "Military standards $810 \mathrm{G}$ test method 513.6 - acceleration." 2014.

[14] C. Piazza, G. Grioli, M. Catalano, and A. Bicchi, "A century of robotic hands," in Ann. Rev. Control, Robotics, and Autonomous System, vol. 2, 2019, pp. 1-32.

[15] W. Friedl, H. Höppner, F. Schmidt, M. A. Roa, and M. Grebenstein, "CLASH: Compliant low cost antagonistic servo hands," in IEEE/RSJ Int. Conf. Intelligent Robots and Systems (IROS), 2018, pp. 6469-6476.

[16] M. C. Carrozza, C. Suppo, F. Sebastiani, B. Massa, F. Vecchi, R. Lazzarini, M. Cutkosky, and P. Dario, "The SPRING hand: development of a self-adaptive prosthesis for restoring natural grasping," Autonomous Robots, vol. 16, no. 2, pp. 125-141, 2004.

[17] C. Gosselin, F. Pelletier, and T. Laliberte, "An anthropomorphic underactuated robotic hand with 15 dofs and a single actuator," in IEEE Int. Conf. on Robotics and Automation (ICRA), 2008, pp. 749-754.

[18] G. Cerruti, D. Chablat, D. Gouaillier, and S. Sakka, "ALPHA: A hybrid self-adaptable hand for a social humanoid robot," in IEEE/RSJ Int. Conf. on Intelligent Robots and Systems (IROS), 2016, pp. 900-906.

[19] R. Deimel and O. Brock, "A novel type of compliant and underactuated robotic hand for dexterous grasping," Int. J. of Robotics Research, vol. 35 , no. 1-3, pp. 161-185, 2016.

[20] B. Hillberry and A. Hall, "US patent 3,932,045," 1976. [Online]. Available: http://www.google.ch/patents/US3932045

[21] C. Eppner, R. Deimel, J. Álvarez Ruiz, M. Maertens, and O. Brock, "Exploitation of environmental constraints in human and robotic grasping," Int. J. Robotics Research, vol. 34, no. 7, pp. 1021-1038, 2015.

[22] J. W. Garrett, "The adult human hand: Some anthropometric and biomechanical considerations," Human Factors, vol. 13, no. 2, pp. 117131, 1971. 